\title{
Evaluation of anemia as a postoperative risk factor in the evolution of patients with gastric resection for malignancies
}

\author{
Bogdan Dumitriu ${ }^{1,2^{*}}$, Sebastian Valcea ${ }^{1,2}$, Gabriel Andrei ${ }^{1,2}$, Mircea Beuran ${ }^{1,2}$ \\ ${ }^{1}$ CAROL DAVILA UNIVERSITY OF MEDICINE AND PHARMACY, BUCHAREST, ROMANIA \\ ${ }^{2}$ CLINICAL EMERGENCY HOSPITAL OF BUCHAREST, DEPARTMENT OF GENERAL SURGERY, BUCHAREST, ROMANIA
}

Introduction. Gastric cancer remains among the top three digestive diseases with the highest mortality rates in the world. Treatment of gastric cancer is multidisciplinary, gastric resection being essential for the best result. Anemia is one of the most common comorbidities present in patients diagnosed with gastric cancer. Materials and Methods. This is a retrospective analytical study over a period of 6 years (2014-2019). It is based on 114 consecutive gastric resections for cancer performed by a single team using exclusively resection and reconstruction stapling methods. The study aims to investigate a correlation between the presence of preoperative anemia and the incidence of postoperative morbidity and mortality. Results. Preoperative anemia was found in $70 \%$ of patients, with about half of these patients presenting with mild anemia. Most postoperative complications were grade I and II according to the Clavien Dindo scale. Anemia was correlated with an increase in infectious complications, anastomotic leaks and secondary peritoneal abscesses, pancreatic complications after multivisceral resection and length of hospital stay. Conclusions. Preoperative anemia is a risk factor that exposes the cancer patient to an increased incidence of life-threatening postoperative complications. In addition, it also extends the length of hospital stay and costs. Therefore, special attention should be paid to the identification and reduction of anemia before extensive gastric surgery in order to obtain the best possible therapeutic result.
\end{abstract}

Category: Original Research Paper
Received: May 04, 2021
Accepted: July 22, 2021
Published: November 15, 2021
Keywords:
anemia, postoperative risk factors, gastric resection,
gastric cancer
*orresponding author:
Bogdan Dumitriu,
Carol Davila University of Medicine and Pharmacy,
Clinical Emergency Hospital of Bucharest, Department
of General Surgery, Bucharest, Romania
E-mail: b_dumitriu@yahoo.com

Category: Original Research Paper

Received: May 04, 2021

Accepted: July 22, 2021

November 15, 202

eywords:

anemia, postoperative risk factors, gastric resection,

orresponding author:

Bogdan Dumitriu of General Surgery, Bucharest, Romania

\section{Introduction}

Gastric cancer remains a leading cause of mortality worldwide, being placed according to the geographical region on the second or third incidence in digestive malignancies (see GLOBOCAN 2020 data) [1].

The treatment of gastric cancer is a continuous challenge, gastric resection being the main treatment option. Gastric surgery involves a variable degree of morbidity and mortality, which is closely dependent on several factors, such as: tumoral characteristics, surgical parameters and the existing comorbidities. One of the most common comorbidities present in patients diagnosed with gastric cancer is anemia. This can be seen as both a comorbidity and a pre-existing risk factor, because, as the results of this study show, it influences the therapeutic outcome of the cancer patient $[2,3]$.
Surgical resection is generally complemented by various oncological treatments. Stage of the disease at the moment of admission represents the most important predictive factor for long term survival of the patient. In addition, many other factors contribute to varying degrees to the outcome of patients operated for gastric malignancies [4].

Generally, the morbidity and mortality associated with gastric cancer surgery are not negligible. Despite the constant improvements of care standards, surgical technologies, dedicated centers and screening procedures, morbidity varies between 9 and $46 \%$, depending on the study. Mortality is around $4-5 \%$, even in tertiary centers with a large number of patients [5-7].

In addition to the stage of the disease at the time of hospitalization, several risk factors/ comorbidities that 
patients may present have proven to be quite important in the evolution and long-term survival.

In this study, we aim to identify a possible correlation between hemoglobin levels in patients prior to surgery, the necessity of blood transfusion during hospitalization and the postoperative evolution in terms of complications in patients undergoing gastric resection for cancer.

\section{Materials and Methods}

This is a retrospective analytical study over a period of 6 years, between 2014 and 2019. It was conducted in the Surgical Clinic of the Emergency Hospital of Bucharest, including 114 patients. Inclusion criteria were represented by patients with a positive diagnosis of gastric cancer, who were operated consecutively by a single team. In addition, the resection and reconstruction procedures were performed exclusively mechanically using surgical staplers. All benign stomach pathologies were excluded from this study.

Most patients were electively hospitalized, but a small group of patients presented with acute upper gastrointestinal bleeding, with or without a prior diagnosis of gastric cancer.

Diagnosis of gastric cancer was done using upper digestive endoscopy with biopsies and histopathological examination. Preoperative staging was performed by computer tomography.

Different types of gastric resections were used, from limited gastrectomy and lymphadenectomy to total gastrectomy with en block resection of multiple organs. The reconstruction phase was performed depending on the resection performed and using the mechanical procedure.

Patients with neoplastic or posthemorrhagic anemia have been identified, and postoperative complications have been classified according to the Clavien Dindo scale.

In the postoperative period, patients were monitored using specific protocols, with daily clinical evaluation, monitoring of vital signs, complete blood tests, monitoring of drainage tubes and urine flow.

Statistical analysis was performed using IBM SPSS version 19 and Microsoft Excel using Fischer test, Student t-test and Pearson correlation.

\section{Results}

The typical profile of the patient included in the study is being represented by males, in the mid 60's from urban settings. Almost three quarters of the patients were males $(73.6 \%)$ with a mean age of 66.5 years.

Gastric carcinoma was the most commonly type of tumor identified. Staging at the time of admission was high in most cases, stage III and IV being predominant (71\%).

Total gastrectomy was the most used surgical procedure (65\%), while the Roux-en-Y esojejunal anastomosis was the most common method of digestive reconstruction performed. Other surgical procedures were also performed, such as distal gastrectomy (20\%), limited wedge resections (10\%) and proximal gastrectomy (5\%) with Billroth I and II reconstructions or esogastric anastomosis.

At admission, about $70 \%$ of patients had a degree of anemia: $36.8 \%$ mild anemia, $21.9 \%$ moderate anemia and $10.5 \%$ severe anemia. The anemia was neoplastic $(55 \%)$ or secondary to upper digestive bleeding (45\%). The majority of patients which presented with upper digestive bleeding reported melena in their history. Furthermore, all patients with severe anemia presented weight loss in the past three months prior to admission.

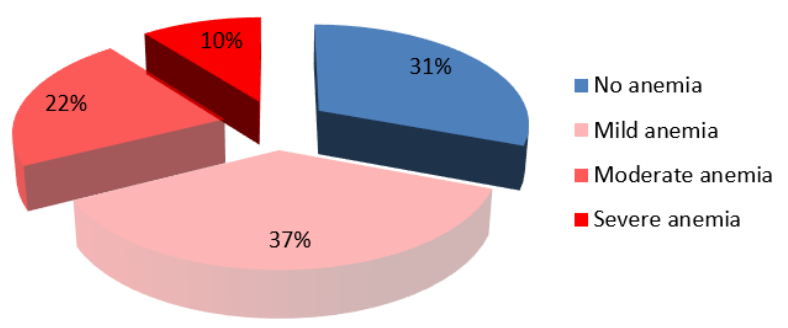

Figure 1. Distribution of patients according to grade of anemia at admission

Postoperatively, we monitored parameters such as hemoglobin level, the need for transfusion, duration of hospitalization, general and specific postoperative complications that were classified according to the Clavien Dindo scale. Specific complications were represented mainly by anastomotic leakage, postoperative hemorrhaging from the stapling lines, peritoneal abscess and functional complications like delayed gastric emptying syndrome, dumping syndrome and anastomotic stricture.

The majority of postoperative complications were grade I and II (30\%), grade III $(23 \%)$, grade IV $(6 \%)$. The mortality rate was $5.98 \%$.

Apart from grade I and II complications, the infectious complications such as surgical site infection, catheter related urinary infections and pneumonia represented the majority cases.

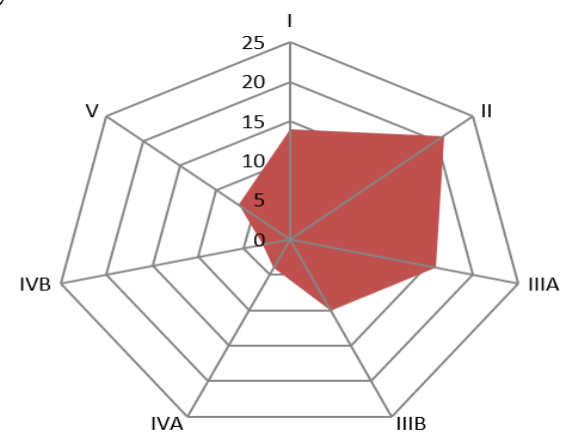

Figure 2. Distribution of postoperative complications according to Clavien Dindo scale 
Preoperative anemia was correlated with the postoperative results obtained. Patients with moderate and severe anemia (hemoglobin levels below $8 \mathrm{~g} / \mathrm{dl}$ ) were found to have an increased incidence of postoperative complications. Using statistical analysis (Pearson correlation), we found that anemic patients were more likely to develop both general and specific complications. Thus, patients with hemoglobin levels below $8 \mathrm{~g} / \mathrm{dl}$ were 3 times more likely to develop anastomotic leakage and subsequently peritoneal abscess or even generalized peritonitis. The general incidence of anastomotic leak was approximately $7 \%$. Six of the eight patients who developed anastomotic leakage were found to have moderate to severe anemia on admission.

Anemia has also been shown to be a risk factor for the development of infectious complications $(\mathrm{r}=0.3288$, $\mathrm{p}=0.002$ ). Of the 21 patients who developed surgical site infections, $15(71.4 \%)$ had a degree of anemia.

Intraperitoneal infections (11\%) were mainly caused by anastomotic leaks. In this subgroup, mortality was up to $20 \%$. Four of the five patients who developed peritoneal abscess in the postoperative period were patients with anemia.

Patients with advanced local gastric cancer (stage III-B and $\mathrm{C}$, with the invasion of the serosa and adjacent organs) required multivisceral en-bloc resection. In most of these cases, distal pancreatectomy and splenectomy were required. This group of patients had an increase in pancreatic complications, manifested as pancreatic leakage (4.2\%) or acute postoperative pancreatitis (5.9\%). These complications were responsible for secondary complications such as peritoneal abscess or multiple organ failure. Mortality in this subgroup was significant, up to $28.5 \%$. Pancreatic complications have been found to be statistically associated with moderate to severe anemia $(\mathrm{r}=0.3168, \mathrm{p}=0.004)$.

The length of hospitalization and, subsequently, the costs were significantly higher for patients with anemia. The average hospitalization of patients without postoperative complications was 7 days. In comparison, patients with anemia who developed different types of complications had an average stay of 21 days. Consequently, there was a significant increase in the length of hospital stay and subsequently the cost in patients with anemia $(\mathrm{p}=0.045)$.

The mortality in the study group was $5.98 \%(n=7)$. Three of the seven patients who died (42.8\%) had anemia at admission; however, no statistically significant correlation was found between anemia and mortality rates. Complications such as acute postoperative pancreatitis $(\mathrm{p}=0.001)$, multiple organ failure $(\mathrm{p}<0.0001)$ and anastomotic leakage $(\mathrm{p}<0.0001)$ have been shown to be factors influencing mortality. Anemia has been shown to be present in most of these complications.
Table 1. Correlations between anemia and different complications

\begin{tabular}{|c|c|c|}
\hline Variable & Pearson & Statistical significance \\
\hline $\begin{array}{c}\text { Infectious } \\
\text { complications }\end{array}$ & 0.3288 & 0.002 \\
\hline $\begin{array}{c}\text { Surgical site } \\
\text { infections }\end{array}$ & 0.2205 & 0.007 \\
\hline $\begin{array}{c}\text { Pulmonary } \\
\text { infections }\end{array}$ & 0.4075 & 0.035 \\
\hline $\begin{array}{c}\text { Anastomotic } \\
\text { leak }\end{array}$ & 0.3255 & 0.0001 \\
\hline $\begin{array}{c}\text { Pancreatic } \\
\text { complications }\end{array}$ & 0.3168 & 0.004 \\
\hline $\begin{array}{c}\text { Multiple organ } \\
\text { dysfunction }\end{array}$ & 0.6822 & $<0.0001$ \\
\hline $\begin{array}{c}\text { Length of } \\
\text { hospital stay }\end{array}$ & 0.4228 & 0.045 \\
\hline
\end{tabular}

\section{Discussion}

Gastric cancer still has a high rate of morbidity and mortality among digestive malignancies [8-11]. The curative treatment of this disease is primarily based on gastric resection with lymphadenectomy. Such surgical procedure is an extensive intervention that requires dedicated surgical teams, with centers that have adequate logistical infrastructure, and a multidisciplinary therapeutic approach. The surgical procedure has rates of morbidity and mortality that cannot be neglected. In addition, these rates are enhanced by various risk factors. Such risk factors can be corrected before the surgery in some cases to get better therapeutic results. One such risk factor is anemia, as suggested by several studies [12-14], especially when the hemoglobin levels is below $8 \mathrm{~g} / \mathrm{dl}$ [15].

This is why we consider important to address gastric cancer in elective patients using two important instruments. The first is screening, which requires a national strategy to be implemented. The second important aspect is the identification of risk factors that can be modulated in the preoperative period. Anemia is easy to identify and should be corrected before surgery in elective patients [16,17].

Studies have shown that the relationship between anemia and an unfavorable prognosis could be linked to several factors. Some authors have suggested that a low hemoglobin level leads to tumor hypoxia which in turn causes resistance to therapy $[15,16]$. Others authors suggested that tumor hypoxia leads to transcription of hypoxia inducible factor (HIF-1), which facilitates tumor growth and aggressiveness [18]. This aggressiveness can be also explained through genetic changes and clonal selection in tumoral cells [19].

We consider that the preoperative treatment of anemia by increasing the hemoglobin level to at least $8 \mathrm{~g} / \mathrm{dl}$ is important in decreasing the rates of postoperative 
morbidity. This can be achieved by implementing the Patient Blood Management into the cancer patient perioperative management protocol [20]. Through this protocol in the preoperative stage, the patient should be checked for the level of hemoglobin, ferritin levels, serum iron and total iron binding capacity. If surgery can be considered elective, a period of 2-3 weeks should be included in the management of anemia by administering iron to patients or even blood transfusions. In this way an important risk factor for the development of postoperative morbidity can be eliminated.

The selection of patients is quite important to obtain a better therapeutic result after gastric resection. Patients diagnosed with gastric cancer who are candidates for elective surgery should be better evaluated in the preoperative period, and should not be considered and treated as a surgical emergency.

Patient Blood Management protocols and ESPEN guidelines require that patients with certain risk factors be properly evaluated and optimized for surgery [20]. Identification of patients with anemia who are not surgical emergencies or who do not present hemodynamic instability are candidates for the correction of anemia with iron products in the preoperative period. In this way, better conditions can be created for the surgical procedure, better therapeutic results can be obtained for patients, and the need for blood transfusions can be minimized.

\section{Conclusions}

Gastric resection remains the gold standard of treatment for oncological pathologies of the stomach. In order to create the best outcome for the patient, tertiary centers with dedicated multidisciplinary teams for treatment of such pathologies are preferred.

Advances in surgical technologies and better intensive care units play a decisive role in reducing possible risks (in terms of morbidity and mortality), thus ensuring the best possible outcomes. Screening programs are vital for the early diagnosis of gastric cancer, and for ensuring curative treatment with prolonged intervals without disease. In addition, we consider that identifying risk factors that can be corrected before surgery is just as important in optimizing the outcome.

Anemia is a complication and also a risk factor that was prevalent in our study group. It has been found to significantly influence morbidity rates, hospitalization and costs. Infectious complications, anastomotic leak and pancreatic complications in multivisceral resections were significantly higher in patients who had anemia prior to surgery. If surgery is considered elective, anemia should be corrected and increased to at least $8 \mathrm{~g} / \mathrm{dl}$.

The approach to gastric cancer needs to be staggered. Identifying potential risk factors and correcting them, if possible, in the preoperative stage is important for lowering morbidity and mortality rates. In this way, the chances of a better therapeutic result are maximized.

\section{Conflict of interest disclosure}

There are no known conflicts of interest in the publication of this article. The manuscript was read and approved by all authors.

\section{Compliance with ethical standards}

Any aspect of the work covered in this manuscript has been conducted with the ethical approval of all relevant bodies and that such approvals are acknowledged within the manuscript.

\section{References}

1. Sung H, Ferlay J, Siegel RL, Laversanne M, Soerjomataram I, Jemal A, Bray F. Global Cancer Statistics 2020: GLOBOCAN Estimates of Incidence and Mortality Worldwide for 36 Cancers in 185 Countries. CA Cancer J Clin. 2021 May;71(3):209249. doi: 10.3322/caac. 21660

2. Liu JY, Peng CW, Yang XJ, Huang CQ, Li Y. The prognosis role of AJCC/UICC 8th edition staging system in gastric cancer, a retrospective analysis. Am J Transl Res. 2018 Jan 15;10(1):292-303.

3. Maehara Y, Orita H, Okuyama T, Moriguchi S, Tsujitani S, Korenaga D, Sugimachi K. Predictors of lymph node metastasis in early gastric cancer. $\mathrm{Br} \mathrm{J}$ Surg. 1992;79(3):245-7. doi: 10.1002/bjs. 1800790320

4. Bartlett EK, Roses RE, Kelz RR, Drebin JA, Fraker DL, Karakousis GC. Morbidity and mortality after total gastrectomy for gastric malignancy using the American College of Surgeons National Surgical Quality Improvement Program database. Surgery. 2014 Aug;156(2):298-304. doi: 10.1016/j.surg.2014.03.022

5. Martin AN, Das D, Turrentine FE, Bauer TW, Adams RB, Zaydfudim VM. Morbidity and Mortality After Gastrectomy: Identification of Modifiable Risk Factors. J Gastrointest Surg. 2016 Sep;20(9):1554-64. doi: 10.1007/s11605-016-3195-y

6. Wang S, Xu L, Wang Q, Li J, Bai B, Li Z, Wu X, Yu $\mathrm{P}, \mathrm{Li} \mathrm{X}$, Yin J. Postoperative complications and prognosis after radical gastrectomy for gastric cancer: a systematic review and meta-analysis of observational studies. World J Surg Oncol. 2019 Mar 18;17(1):52. doi: 10.1186/s12957-019-1593-9

7. Papenfuss WA, Kukar M, Oxenberg J, Attwood K, Nurkin S, Malhotra U, Wilkinson NW. Morbidity and mortality associated with gastrectomy for gastric cancer. Ann Surg Oncol. 2014 Sep;21(9):3008-14. doi: 10.1245/s10434-014-3664-Z

8. Jee YS, Hwang SH, Rao J, Park DJ, Kim HH, Lee HJ, Yang HK, Lee KU. Safety of extended endoscopic 
mucosal resection and endoscopic submucosal dissection following the Japanese Gastric Cancer Association treatment guidelines. Br J Surg. 2009 Oct;96(10):1157-61. doi: 10.1002/bjs.6686

9. Smyth EC, Verheij M, Allum W, Cunningham D, Cervantes A, Arnold D; ESMO Guidelines Committee. Gastric cancer: ESMO Clinical Practice Guidelines for diagnosis, treatment and follow-up. Ann Oncol. 2016; 27(suppl 5):v38-v49. doi: 10.1093/annonc/mdw350

10. Slavu I, Alecu L, Tulin A, Stanculeanu DL, Nitipir C. Laparoscopic resection of gastric GISTs. Where do we stand now? A single-centered experience. J Mind Med Sci. 2019;6(2):334-339. doi: 10.22543/7674.62.P334339

11. Uzun O, Senger AS, Gülmez S, Ömeroğlu S, Ofluoğlu CB, Öz A, Polat E, Duman M. Evaluating the effect of tumor size on survival and its prognostic significance among gastric cancer patients. J Clin Invest Surg. 2020;5(2):76-82. doi: 10.25083/2559.5555/5.2/76.82

12. Huang XZ, Yang YC, Chen Y, Wu CC, Lin RF, Wang $\mathrm{ZN}$, Zhang $\mathrm{X}$. Preoperative Anemia or Low Hemoglobin Predicts Poor Prognosis in Gastric Cancer Patients: A Meta-Analysis. Dis Markers. 2019 Jan 2;2019:7606128. doi: 10.1155/2019/7606128

13. Liu X, Qiu H, Huang Y, Xu D, Li W, Li Y, Chen Y, Zhou Z, Sun X. Impact of preoperative anemia on outcomes in patients undergoing curative resection for gastric cancer: a single-institution retrospective analysis of 2163 Chinese patients. Cancer Med. 2018 Feb;7(2):360-369. doi: 10.1002/cam4.1309

14. Shen JG, Cheong JH, Hyung WJ, Kim J, Choi SH, Noh SH. Pretreatment anemia is associated with poorer survival in patients with stage I and II gastric cancer. $J$ Surg Oncol. 2005 Aug 1;91(2):126-30. doi: $10.1002 /$ jso. 20272
15. Li WH, Zhang JY, Liu WH, Chen XX. Role of the initial degree of anaemia and treatment model in the prognosis of gastric cancer patients treated by chemotherapy: a retrospective analysis. BMC Cancer. 2020 May 13;20(1):414. doi: 10.1186/s12885-02006881-7

16. Toprak SS, Gultekin Y. A combination of laparoscopic Nissen fundoplication and laparoscopic gastric plication for gastric esophageal reflux disease and morbid obesity. J Clin Invest Surg. 2019;4(2):81-87. doi: 10.25083/2559.5555/4.2/81.87

17. Dumitru A, Nitipir C, Preda G, Marcu DR, Badiu DC, Andreea G, Pop CS, Serban LM, Hulea R, Pituru S. Ethical aspects in managing patients diagnosed with digestive cancers; a review of literature. J Mind Med Sci. 2019;6(1):1-5. doi: 10.22543/7674.61.P15

18. Vaupel P. The role of hypoxia-induced factors in tumor progression. Oncologist. 2004;9 Suppl 5:10-7. doi: 10.1634/theoncologist.9-90005-10

19. Dachs GU, Tozer GM. Hypoxia modulated gene expression: angiogenesis, metastasis and therapeutic exploitation. Eur J Cancer. 2000 Aug;36(13 Spec No):1649-60. doi: 10.1016/s0959-8049(00)00159-3

20. Filipescu D, Bănăţeanu R, Beuran M, Burcoş T, Corneci D, Cristian D, Diculescu M, Dobrotă A, Droc G, Isacoff D, Goşa D, Grinţescu I, Lupu A, Mirea L, Posea C, Stanca O, Ştefan M, Tomescu D, Tudor C, Ungureanu D, Mircescu G. Perioperative Patient Blood Management Programme. Multidisciplinary recommendations from the Patient Blood Management Initiative Group. Rom J Anaesth Intensive Care. 2017 Oct;24(2):139-157. doi: 10.21454/rjaic.7518.242.fil 